\title{
PALEO
}

Revue d'archéologie préhistorique

19 | 2007

Spécial table ronde (1 ère partie) : Le Gravettien : entités régionales d'une paléoculture européenne, Les Eyzies, juillet 2004

\section{Traits essentiels du Gravettien en Italie}

Main features of Gravettian in Italy

\section{Paolo Gambassini}

\section{(2) OpenEdition}

\section{Journals}

Édition électronique

URL : https://journals.openedition.org/paleo/542

DOI : $10.4000 /$ paleo.542

ISSN : 2101-0420

Éditeur

SAMRA

Édition imprimée

Date de publication : 30 décembre 2007

Pagination : 105-108

ISSN : 1145-3370

Référence électronique

Paolo Gambassini, «Traits essentiels du Gravettien en Italie », PALEO [En ligne], 19 | 2007, mis en ligne le 23 avril 2009, consulté le 30 juin 2021. URL : http://journals.openedition.org/paleo/542 ; DOI :

https://doi.org/10.4000/paleo.542

\section{(ब) $\odot \Theta$}

PALEO est mis à disposition selon les termes de la licence Creative Commons Attribution - Pas d'Utilisation Commerciale - Pas de Modification 4.0 International. 


\title{
TRAITS ESSENTIELS DU GRAVETTIEN EN ITALIE
}

\author{
Paolo GAMBASSINI ${ }^{(1)}$
}

\begin{abstract}
Résumé : En comptant sur une vingtaine de sites gravettiens en Italie, on observe que, en partant d'un faciès commun à pointes à dos (28-27 Ky BP), les deux versants de la péninsule montrent un différent développement. Le faciès Noailles est limité au versant tyrrhénien, tandis qu'à l'Est on trouve des complexes à dos tronqués. Les Apennines se posent comme une sorte de frontière entre les Gravettiens provenant de France et ceux provenant de l'Europe orientale.
\end{abstract}

Mots-clés : Paléolithique, Gravettien, Italie.

Abstract: Main features of Gravettian in Italy. According to what has been observed on about twenty gravettian sites known in the country, Italian Gravettian begins with a single facies, dated around 28-27 Ky BP and characterised by backed points. Afterwards, two separate cultures appear along sides of the country: Noailles culture is limited on Tyrrhenian coastline, while eastern side contains lithic assemblages characterised by backed truncated pieces. Apennines constituted a borderline between gravettian people coming from France and the ones from Eastern Europe.

Key-words : Palaeolithic, Gravettian, Italy.

La présence gravettienne en Italie est attestée sur une vingtaine de sites dont la liste correspond pratiquement à celle indiquée par A. Palma di Cesnola (1993) dans son ouvrage de synthèse sur le Paléolithique supérieur italien. L'importance scientifique de ces sites est très variable puisque l'on trouve des gisements stratifiés dans des grottes ou sous abri, mais aussi en plein air, où l'industrie gravettienne se ramasse en surface, parfois mélangée à des éléments d'autres périodes. Si ces derniers sites se traduisent en points sur une carte, comme simples témoins d'une présence, se sont les séries stratigraphiques qui fournissent les informations culturelles, chronologiques et paléoenvironnementales nécessaires.

Nous pouvons ajouter à la liste de Palma, qui comptait dixhuit sites gravettiens, deux sites liés à des recherches récentes : la grotte de la Serratura à Camerota et le site de plein air de Bilancino, près de Florence.

Du point de vue géographique, on observe que les stations gravettiennes sont, pour la plupart, distribuées sur le versant tyrrhénien, alors que les sites à l'est des Appennins sont rares. Les séries de référence qui permettent d'évaluer divers faciès gravettiens, sont celles de Riparo Mochi en Ligurie, de la grotte della Cala en Campanie et de la grotte Paglicci dans les Pouilles.
Ces séries indiquent que le premier peuplement gravettien en Italie correspond au faciès "à pointes à dos", selon la définition de Palma di Cesnola (2004), équivalent au Gravettien "indifférencié" de Laplace. Ce faciès, en raison de l'absence d'aspects particuliers ou de "fossiles directeurs", est caractérisé par une abondance de pointes à dos, la plupart de très petite taille. La tendance à la fabrication de microgravettes atteint son maximum à la grotte della Cala, où les pointes aciculaires de 10-12 mm de long sont fréquentes, parfois produites sur des chutes de burin. A Paglicci, le microlithisme est évalué en relation avec le territoire local, riche en silex; même s'il y a abondance de microgravettes, celles-ci n'atteignent pas les dimensions des plus petites microgravettes de la grotte della Cala.

On peut souligner, dans ce premier Gravettien, quelques traits aurignacoïdes représentés par des grattoirs carènes, des museaux, des burins carénés et des lamelles (Cala) à retouche demi-abrupte proches de celles du type Dufour (Boscato et al. 1997).

En ce qui concerne la chronologie radiométrique, l'industrie en apparence la plus ancienne est celle de Paglicci (niveau 23), datée de $28100 \pm 400 \mathrm{BP}$, alors que le niveau GB3 de la Cala, le plus ancien de la séquence gravettienne locale, est daté de $26880 \pm 320$. 


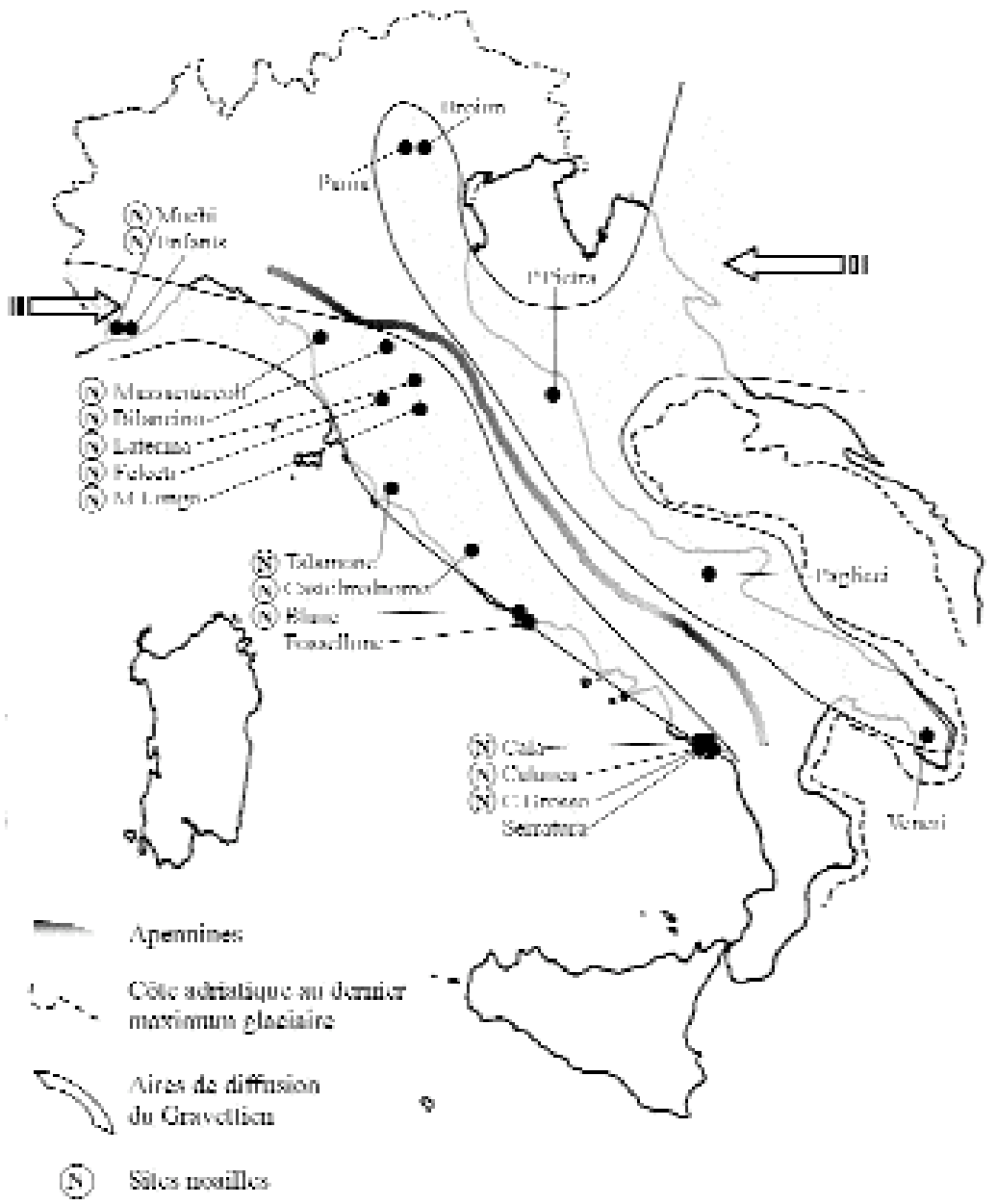

Figure : Localisation des sites gravettiens en Italie.

Figure: Location of gravettian sites in Italy.

Les niveaux attribuables au même faciès indifférencié, de l'abri Mochi et de la Calanca (cette demière de la même aire que celle de la Cala) ne sont pas datés.

Si ces dates, encore trop peu nombreuses, devaient être confirmées, nous devrions supposer que la première diffusion du Gravettien en Italie a eu lieu par une porte orientale. L'absence presque totale de dates dans l'aire balkanique ne permet pas, pour le moment, de formuler des hypothèses sur la trajectoire de diffusion des populations gravettiennes mais on peut présumer, au moins pour la portion transadriatique, qu'un rôle important a été joué par le tracé de la côte, ainsi que par la plaine côtière, située bien plus au sud que sa situation actuelle à cause de la régression glacioeustatique (Vai et Cantelli eds. 2004).

Plusieurs indices présents sur les sites des Pouilles témoignent en faveur de la provenance orientale, en particulier à la grotte Paglicci et à la grotte des Vénus de Parabita.

A Paglicci, sur les sites gravettiens, on trouve :

- quelques couteaux de Kostienki, absents dans le reste de l'Italie ;

- la sépulture féminine PA25, datée d'il y a environ 23000 ans, avec diadème en dents percées rapportables aux modèles est-européens (Palma di Cesnola 2004) ;
- la sépulture PA12, datée d'il y a environ 24700 ans, dont l'ADN mitocondrial a des caractères en commun avec celui des populations du Proche Orient et du Caucase (Caramelli et al. 2003).

Deux exemplaires de Vénus en os, que l'on peut confronter à des figures analogues de Kostienki (Radmilli 1966), proviennent de Parabita.

La porte orientale, déjà supposée dans le passé par Mochi et plus récemment évoquée par Palma di Cesnola (1993), doit avoir longtemps permis le passage des Gravettiens vers I'Italie de l'est. En effet, si l'on se fie aux dates ${ }^{14} \mathrm{C}$, on passe de 28000 ans BP environ de la base de la série stratigraphique de Paglicci, à 20000 ans BP environ avec les industries finales de la période. Durant cet arc chronologique, le faciès de l'industrie lithique présente deux changements fondamentaux : au Gravettien antique à pointes à dos, se substitue d'abord un Gravettien évolué avec microgravettes et de rares pointes de La Font-Robert et puis un Gravettien final à dos anguleux. II est plausible que le flux de populations d'Orient n'ait pas eu lieu de manière continue mais, au contraire, par pulsations successives qui ont laissé des traces dans la séquence stratigraphique de Paglicci. 
La situation sur la façade occidentale de la péninsule, où les sites gravettiens sont les plus nombreux, est très différente avec des faciès presque exclusivement à burins de Noailles. Si l'on prend par exemple la série stratigraphique de la Cala, nous constatons qu'initialement, à une date proche de 27000 ans BP, on trouve un niveau gravettien indifférencié - avec des caractères en partie semblables à ceux de Paglicci - qui a comme trait caractéristique une dimension microlithique des armatures à dos (Boscato et al. 1997 ; Borgia et Wierer 2005). Le changement de faciès suivant intervient aux alentours de 25000 ans (OxA 6263 $24620 \pm 220$ ), avec l'avènement d'industries à burins de Noailles ; ces derniers se rarérifiant par rapport aux industries analogues plus septentrionales. L'hypothèse de Palma di Cesnola (1993) est encore valable, selon laquelle une migration le long de la zone côtière tyrrénienne, partant de la France, arrive, en l'état actuel, jusqu'au Cilento, alors que la présence des produits manufacturés les plus caractéristiques et diagnostiques du faciès diminue progressivement.

Il est peut-être prématuré de se demander ce qui a bien pu donner le coup d'envoi à la diffusion des populations "Noailles " vers des latitudes plus basses par rapport à l'aire française ; aux causes généralement invoquées pour des phénomènes similaires, comme la pression démographique et le changement environnemental, nous pouvons ajouter un nouvel élément de réflexion. II s'agit de l'hypothèse, due à de récentes recherches (Aranguren et Revedin 2001, 2005), de l'association entre le burin de Noailles et le travail des herbes palustres. Dans ce cas, la diffusion devrait être vue comme une réponse à un facteur environnemental à mettre en relation avec la présence de matières particulièrement recherchées par les Gravettiens de l'époque.

La série de la Cala ne contient pas d'informations sur les niveaux gravettiens plus récents que ceux à burins de Noailles, à cause d'une importante lacune stratigraphique, avec un trou de plusieurs milliers d'années après lesquels se dépose une couche d'Epigravettien évolué. Un tel vide est en partie comblé par la série de la grotte voisine de la Serratura où une couche avec industrie gravettienne à dos tronqués datée des alentours de 20000 ans BP (Martini et al. 2001) se superpose à un niveau à burins de Noailles.

La différence entre les deux versants de la péninsule est donc considérable et il n'y a pas de preuves, en l'état actuel des recherches, de contacts entre un versant et l'autre. Nous devons penser que la chaîne des Appennins a découragé son franchissement par différents groupes gravettiens (figure), dans un sens ou dans l'autre.

A première vue, l'hypothèse semble faible, puisque les Appennins présentent aujourd'hui encore des cols à des altitudes relatives basses et les témoignages glaciaires en Italie centrale et méridionale permettent d'exclure que même lors des périodes les plus froides, il y ait eu des glaciers en mesure de constituer un obstacle au franchissement de la chaîne.

Cependant, il est vrai qu'au cours d'autres moments du Paléolithique, on observe également des différences sen- sibles entre l'aire adriatique et l'aire tyrrénienne. En définitive, les Appennins ont fonctionné comme une barrière même si celle-ci n'était pas en fait insurmontable.

Nous pouvons alors nous demander si une chaîne montagneuse modeste est suffisante pour arrêter un flux de population paléolithique et quel aura été le rôle des Balkans par rapport à l'hypothèse citée de la diffusion de certains groupes gravettiens à partir de l'Orient ? En voulant rester sur cette hypothèse, comme cela nous semble juste, nous devrons chercher des voies de pénétrations directes, à travers l'aire montagneuse balkanique, ou bien formuler l'hypothèse d'un parcours de diffusion plus long, sur la façade côtière méditerranéenne.

Les données jusqu'ici disponibles sont tout à fait insuffisantes pour affronter le problème ; nous pouvons seulement espérer en de futures recherches.

\section{BIBLIOGRAPHIE}

ARANGUREN B., REVEDIN A. 2001 - Interprétation fonctionnelle d'un site à burins de Noailles, L'Anthropologie, 105, pp. 533-545.

ARANGUREN B., REVEDIN A. 2005 - Dalla tipologia analitica alla catena operativa e funzionale: per una nuova definizione del bulino di Noailles, Miscellanea in memoria di Georges Laplace, Rivista di Scienze Preistoriche, supplemento 1, pp. 137-149.

BORGIA V., WIERER U. 2005 - Le punte a dorso degli strati alla base della serie gravettiana di Grotta della Cala (Marina di Camerota, Salemo), Miscellanea in memoria di Georges Laplace, Rivista di Scienze Preistoriche, supplemento 1, pp. 121-135.

BOSCATO P., RONCHITELLI A., WIERER U. 1997 - II Gravettiano antico della Grotta della Cala a Marina di Camerota. Paletnologia e ambiente, Rivista di Scienze Preistoriche, vol. 48, pp. 97-186

CARAMELLI D., LALUEZA-FOX C., VERNESI C., LARI M., CASOLI A., MALLEGNI F., CHIARELLI B., DUPANLOUP I., BERTRANPETIT J., BARBUJANI G., BERTORELLE G. 2003 - Evidence for a genetic discontinuity between Neandertals and 24000-year-old anatomically modern Europeans, Proceedings of National Academy of Science of U.S.A., Maggio 27, 100 (11), pp. 6593-7

MARTINI F., DI CARLO R., MARTINO G. 2001 - Una nuova facies gravettiana sul versante tirrenico a Grotta della Serratura, Rivista di scienze Preistoriche, vol. 51, pp. 139-175

PALMA di CESNOLA A. 1993 - // Paleolitico superiore in Italia, Garlatti e Razzai ed., Firenze, pp. 574

PALMA di CESNOLA A. 2004 - Paglicci. L'Aurignaziano e il Gravettiano antico, Grenzi ed., Foggia, pp. 212 
PISCOPO G., RADMILLI M. 1966 - Sul rinvenimento di due veneri paleolitiche a Parabita (Lecce), Atti della Società Toscana di Scienze Naturali, Serie A, vol. 73, fascicolo 1, pp. $148-156$
RADMILLI M. 1966 - Le due "Veneri" di Parabita, Rivista di scienze Preistoriche, vol. 21, pp. 123-133

VAI G.B., CANTELLI L. (eds.) 2004 - Climex Maps Italy, Map 1- Last Glacial Maximum, ENEA, Roma. 